\title{
Correlation of liver parenchymal gadolinium-ethoxybenzyl diethylenetriaminepentaacetic acid enhancement and liver function in humans with hepatocellular carcinoma
}

\author{
KEI KUBOTA, TAIJI TAMURA, NOBUTAKA AOYAMA, MUNENOBU NOGAMI, \\ NORIHIKO HAMADA, AKIHITO NISHIOKA and YASUHIRO OGAWA
}

Department of Radiology, Kochi Medical School, Kochi University, Kohasu, Oko-Cho, Nankoku, Kochi 783-8505, Japan

Received November 14, 2011; Accepted January 23, 2012

DOI: $10.3892 / \mathrm{ol} .2012 .629$

\begin{abstract}
Animal studies have demonstrated that liver function parameters affect the degree of liver enhancement by gadolinium-ethoxybenzyl diethylenetriaminepentaacetic acid (Gd-EOB-DTPA). The present study prospectively investigated whether liver function parameters and liver damage scores similarly correlate with the degree of liver enhancement by Gd-EOB-DTPA in humans with hepatocellular carcinoma (HCC). A total of 41 patients (32 males, 9 females; mean age, 71.9 years; range, 38-86 years) with suspected HCC provided written, informed consent to undergo a Gd-EOB-DTPA (30 $\mu \mathrm{mol} / \mathrm{kg}$ of body weight)-enhanced T1-gradient-echo (GRE) magnetic resonance imaging (MRI) study. The signal intensity of the liver parenchyma was quantified at various time points following injection of Gd-EOB-DTPA. We investigated the correlations between maximal relative enhancement (RE) values and liver function parameters, and liver damage scores. Correlations between parameters and maximum RE values were determined using the Student's t-test and univariate regression analyses. The effect of potential confounding factors was controlled by multiple stepwise regression analysis. Two-tailed values of $\mathrm{p}<0.05$ were considered to indicate a statistically significant difference. The RE values were maximal in 8 and 33 patients at 20 and $30 \mathrm{~min}$, respectively, following Gd-EOB-DTPA injection and did not significantly differ between respective liver damage scores. Univariate analyses revealed that maximal $R E$ values were associated with serum aspartate aminotransferase, total bilirubin, albumin and 15-min indocyanine green retention rates. Multiple stepwise regression analyses revealed that
\end{abstract}

Correspondence to: Dr Kei Kubota, Department of Radiology, Kochi Medical School, Kochi University, Kohasu, Oko-Cho, Nankoku-Shi, Kochi 783-8505, Japan

E-mail: kubotak@kochi-u.ac.jp

Key words: gadolinium-ethoxybenzyl diethylenetriaminepentaacetic acid, magnetic resonance imaging, contrast material, liver function, hepatocellular carcinoma serum albumin and total bilirubin remained independently significant. The degree of liver parenchyma enhancement by Gd-EOB-DTPA depends on liver function parameters in humans, as in animals. The results from this study suggest that Gd-EOB-DTPA has potential for use as a liver function test, and for providing a short examination time for liver MRI results in patients with normal liver function.

\section{Introduction}

The liver-specific magnetic resonance imaging (MRI) contrast agent gadolinium-ethoxybenzyl diethylenetriaminepentaacetic acid (Gd-EOB-DTPA, Primovist ${ }^{\circledR}$; Bayer Schering Pharma, Berlin, Germany) is used to detect focal liver lesions (1-10) and evaluate the biliary tree $(11,12)$. Intravenously injected Gd-EOB-DTPA is gradually absorbed by hepatocytes and finally excreted via the biliary tract. As a result of hepatocyte uptake, normal liver parenchyma exhibit T1 shortening, unlike focal liver lesions, including those caused by hepatic metastasis. Certain experimental animal studies have demonstrated that this contrast material has the potential to evaluate liver function or detect diffuse liver disease (13-18). Experimentally induced hepatic dysfunction decreases the degree of liver enhancement produced by Gd-EOB-DTPA and prolongs the washout of contrast material $(17,18)$. In rats, liver enhancement with Gd-EOB-DTPA during MRI is delayed and prolonged following liver transplantation as compared with controls (14). The correlation between liver function and Gd-EOB-DTPA kinetics has been evaluated in animal models, but never in humans. The livers of patients with liver tumors are occasionally damaged, which might degrade the contrast between liver and lesion produced by the decreased hepatocyte uptake of Gd-EOB-DTPA. The present study investigated the correlation between hepatic function and liver parenchymal enhancement in Gd-EOB-DTPA-enhanced MRI.

\section{Materials and methods}

Patient population. The present study was performed in accordance with the recommendations of the Declaration of Helsinki. All patients gave their written, informed consent upon enrollment prior to undergoing MRI. In total, 

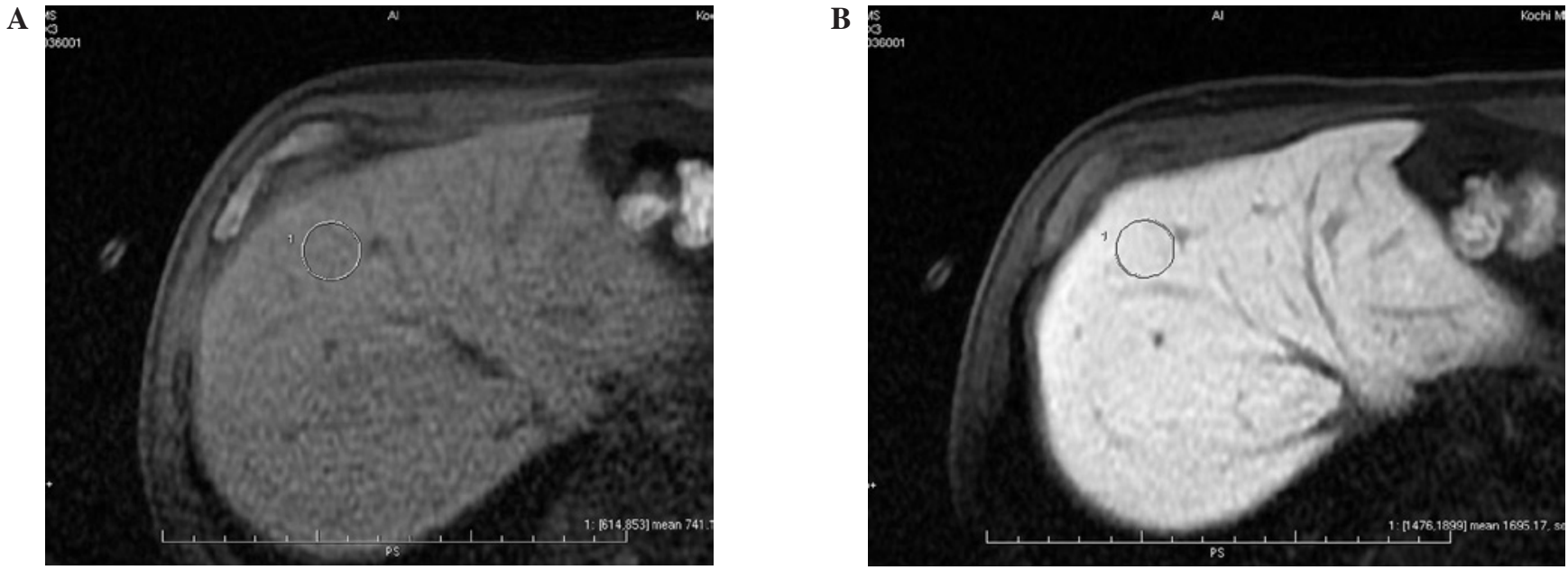

Figure 1. T1-weighted-GRE images at (A) the pre-contrast and (B) hepatocyte uptake phase (20 min) from a 62-year-old man with hepatocellular carcinoma. The location of the region of interest was maintained as constant as possible for each patient at different time points. GRE, gradient echo.

49 consecutive patients with chronic hepatitis were referred to the Interventional Radiology unit at our institution between March 2, 2008 and June 30, 2009 for curative treatment of hepatocellular carcinoma (HCC). All patients except those with MRI contraindications $(n=2)$, including claustrophobia and presence of a pacemaker, underwent Gd-EOB-DTPA-enhanced MRI prior to receiving treatment for HCC. Six (12\%) of the 49 patients were considered ineligible for the study due to multifocal HCC (defined as the presence of $>10$ tumor nodules, $\mathrm{n}=5$ ), or inadequate MRI results (unacceptable image quality due to motion artifacts, $n=1$ ). The remaining 41 patients who were able to undergo MRI and who met the study criteria (mean age, 71.9 years; range, 38-78 years) comprised the study cohort, which included 32 males (mean age, 73.5 years; range, 59-86) and 9 females (mean age, 65.9 years; range, 38-78). Three patients had hepatitis B, 36 had hepatitis C, and two had alcohol-related hepatitis.

MRI. MR images were obtained using a 1.5-T system (Signa HDx; GE Medical Systems, Milwaukee, WI, USA) with an eight-channel anteroposterior phased-array surface coil placed around the patient and covering the entire liver. Imaging protocols included unenhanced sequences [transaxial T2-weighted fast spin-echo (FSE) and in- and opposed-phase GRE sequences] and Gd-EOB-DTPA-enhanced dynamic three-dimensional (3D)-GRE sequences. Images were captured in the transverse plane during an end-expiratory breath-hold with a combined eight-coil element, anteroposterior phased-array surface coil. Using parallel imaging with sensitivity encoding by a factor of two, the total acquisition time was decreased to approximately $15 \mathrm{sec}$. A three-quarter field-of-view was used in the phase-encoding direction. Presaturation pulses were applied above and below the imaging volume to diminish flow artifacts. The patients were administered a $30 \mu \mathrm{mol} / \mathrm{kg}(0.12 \mathrm{ml} / \mathrm{kg}$ body weight $)$ dose of Gd-EOB-DTPA (Primovist) via the antecubital vein at a rate of $2 \mathrm{ml} / \mathrm{s}$ through a 22-gauge intravenous catheter using a power injector (Spectris Solaris ${ }^{\circledR}$ EP; Medrad, Indianola, PA, USA), followed by a $40 \mathrm{ml}$ saline flush at the same injection rate. Dynamic and delayed images were obtained using a fat-suppressed 3D T1-weighted GRE sequence with parallel imaging [LAVA ${ }^{\mathrm{TM}}$ EFGRE ASSET ${ }^{\mathrm{TM}}$ breath-hold; repetition time/echo time (TR/TE), 3.0/1.6 msec; flip angle, $15^{\circ}$; field of view, $42 \times 42 \mathrm{~cm}$; matrix, $384 \times 256$ interpolated to $512 \times 512$; thickness, $5 \mathrm{~mm}$; overlap $2 \mathrm{~mm}$, ASSET acceleration, 2.0]. T1-weighted dynamic GRE breath-hold images were captured at 30 and $180 \mathrm{sec}$ following contrast material administration during the hepatic arterial dominant and equilibrium phases, respectively, and during the delayed hepatobiliary phase at 10 , 20 and 30 min following injection.

Quantitative image analysis. Two radiologists obtained signal intensity values for the liver using a monitor-defined region of interest ( $>50$ pixels) while avoiding major intrahepatic vessels. The location of the region of interest was maintained as constant as possible for individual patients at different time points (Fig. 1). Relative enhancement (RE) of the liver was calculated using the equation: $\mathrm{RE}=\mathrm{SI}_{\text {postcontrast }} / \mathrm{SI}_{\text {precontrast }}$ where $\mathrm{SI}_{\text {precontrast }}$ is the signal intensity of the liver on the precontrast image, and $\mathrm{SI}_{\text {postcontrast }}$ is the signal intensity of the liver on the postcontrast image.

Liver function parameters. Blood serum parameters (total bilirubin, serum albumin and international normalized ratio of prothrombin time), the $15 \mathrm{~min}$ retention rate of indocyanine green test (ICG-R15 test; Daiichi-Sankyou, Tokyo, Japan) and the presence or absence of ascites were recorded to evaluate the degree of liver damage 1-5 days prior to undergoing Gd-EOB-DTPA-enhanced MRI. The degree of liver damage was assessed according to a system similar to the Child-Pugh classification except for inclusion of the ICG test; this evaluation procedure followed the algorithm for HCC treatment guidelines in Japan (19). Aspartate aminotransferase (AST) and alanine aminotransferase (ALT) were also recorded, as these parameters correlated with the degree of liver enhancement in animal studies using Gd-EOB-DTPA-enhanced MRI. Since all 41 patients included were referred from the Interventional Radiology unit, and MRI was part of the workup prior to administering treatment for HCC, these laboratory investigations were undertaken for clinical reasons. 
Table I. Demographic data of the study subjects $(n=41)$.

\begin{tabular}{lr}
\hline Factors & Mean \pm SD \\
\hline Age (years) & $71.9 \pm 9.31$ \\
AST (IU/l) & $45.1 \pm 24.8$ \\
ALT (IU/l) & $37.8 \pm 21.3$ \\
Total bilirubin & $0.85 \pm 0.32$ \\
Serum albumin & $3.8 \pm 0.43$ \\
ICG R15 (\%) & $26.5 \pm 13.2$ \\
Prothrombin time $(\%)$ & $77.9 \pm 12.8$
\end{tabular}

SD, standard deviation; AST, aspartate aminotransferase; ALT, alanine aminotransferase; ICG R15 (\%), 15-min retention rate of indocyanine green test.

Blood serum parameters, as well as the degree of liver damage, were compared with the quantitative parameter of liver enhancement observed on the Gd-EOB-DTPA images.

Statistical analysis. Data were statistically analyzed using the SPSS software package version 11.0 for Windows (SPSS Inc., Chicago, IL, USA). Continuous variables are presented as the mean and standard deviation (SD) as appropriate. The Student's t-test was used to compare RE values among respective liver damage scores. Correlations between the continuous variables and the RE values were determined using univariate regression analyses. Independent determinants of $R E$ values were determined by forward multiple stepwise regression analyses. In the two-tailed test, $\mathrm{P}<0.05$ was considered to indicate a statistically significant difference.

\section{Results}

Time profile of $R E$ values. Fig. 2 shows the time profile of $\mathrm{RE}$ values for the respective liver damage scores. Following injection of Gd-EOB-DTPA, T1-weighted 3D-GRE images revealed an early contrast effect in the liver parenchyma, with a steep increase in signal intensity at $30 \mathrm{sec}$. A further, although slower, increase for up to approximately $20 \mathrm{~min}$ following injection was followed by a plateau of enhancement in the two groups with liver damage. The maximal $\mathrm{RE}$ value was selected for each patient ( 8 and 33 patients at 20 and 30 min, respectively). Maximal RE values did not significantly differ between liver damage levels A and B. None of the study population had ascites or level $\mathrm{C}$ liver damage.

Correlation between RE values and liver function parameters. Table I provides patient demographic data, and Table II shows the results of univariate and multivariate analyses for determinants of maximal RE values. The parameters that significantly related to maximal $R E$ values were serum albumin $(r=0.496$, $\mathrm{p}=0.001)$, AST $(\mathrm{r}=-0.366, \mathrm{p}=0.023)$, total bilirubin $(\mathrm{r}=-0.487$, $\mathrm{p}=0.002)$ and ICG-R15 ( $\mathrm{r}=-0.462, \mathrm{p}=0.003)$. Multiple stepwise regression analysis revealed that serum albumin $(\mathrm{p}=0.002)$ and total bilirubin $(\mathrm{p}=0.001$, inversely) among these parameters remained significantly (Table II) and independently related to maximal $R E$ values $(\mathrm{R} 2=0.287)$.
Table II. Determinants of maximal relative enhancement values by stepwise regression (forward selection).

\begin{tabular}{lcccccc}
\hline & \multicolumn{3}{c}{ Univariate $^{\mathrm{a}}$} & & \multicolumn{4}{c}{ Multivariate $^{\mathrm{b}}$} \\
\cline { 2 - 4 } \cline { 6 - 8 } Factors & $\beta$ & $\mathrm{p}$ & & $\beta$ & $\mathrm{F}$ & $\mathrm{p}$ \\
\hline Age (years) & -0.002 & $\mathrm{NS}$ & & - & - & - \\
AST (IU/l) & -0.366 & 0.023 & & - & - & - \\
ALT (IU/l) & -0.002 & $\mathrm{NS}$ & - & - & - \\
Total bilirubin & -0.487 & 0.002 & -0.285 & 4.169 & 0.001 \\
Serum albumin & 0.496 & 0.001 & 0.224 & 4.61 & 0.002 \\
ICG R15 (\%) & -0.462 & 0.003 & & - & - & - \\
Prothrombin & 0.004 & $\mathrm{NS}$ & & - & - & - \\
time (\%) & & & & 0.287 & - & - \\
R2 & - & - & 0. & & \\
\hline
\end{tabular}

${ }^{\mathrm{a}}$ Univariate coefficients, ${ }^{\mathrm{b}}$ Stepwise multivariate regression analysis was performed. $\beta$ indicates regression coefficient; F, F value of the overall regression model; NS, not significant; ICG R15 (\%), 15-min retention rate of indocyanine green test; AST, aspartate aminotransferase; ALT, alanine aminotransferase.

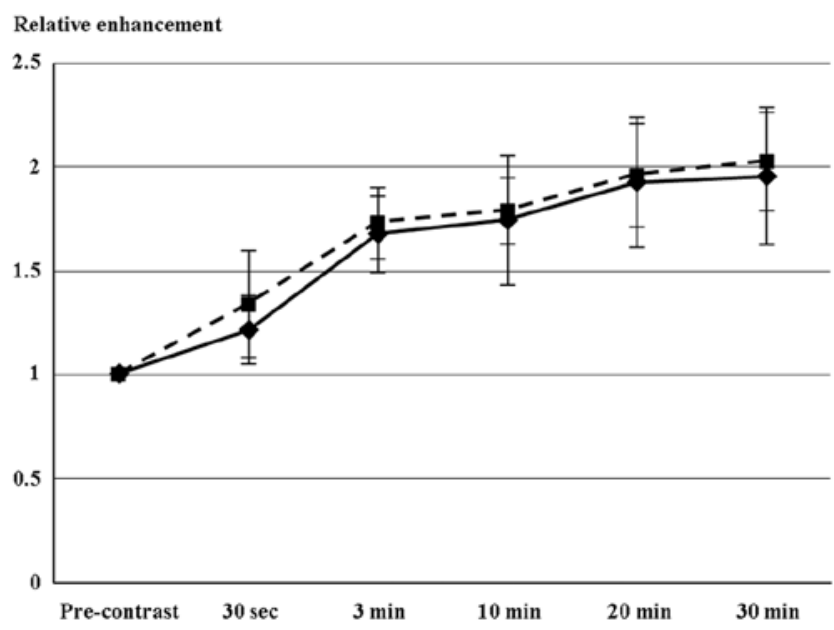

Figure 2. Time profile of the mean relative enhancement following administration of Gd-EOB-DTPA. The error bars are the standard deviation. The solid and dotted lines show liver damage levels A and B, respectively. Gd-EOBDTPA, gadolinium-ethoxybenzyl diethylenetriaminepentaacetic acid.

\section{Discussion}

Our findings demonstrate that the degree of liver enhancement by Gd-EOB-DTPA during the hepatic uptake phase in humans depends on liver function. Certain experimental animal studies have described the potential of Gd-EOB-DTPA to evaluate liver function and diffuse hepatic disease (15-18). Hepatocyte uptake and biliary excretion of Gd-EOB-DTPA, bilirubin and ICG appear to be mediated by glutathione-s-transferase (20-22). Kim et al (17) reported that Gd-EOB-DTPA enhancement in animal livers with chemically induced hepatitis correlates with plasma bilirubin level and ICG clearance. Our findings in patients with hepatitis confirmed the results of Kim et al and the physiological mechanism of Gd-EOB-DTPA. The 
prognosis of patients with cirrhosis and HCC depends on residual liver function and tumor extension $(23,24)$. Various prognostic staging systems for HCC combine the Child-Pugh liver function classification with tumor extension $(23,24)$. The preoperative assessment of functional reserve is significant for estimating the extent of hepatectomy. Hepatic functional reserve is widely assessed using the ICG test, but technetium-99m-galactosyl human serum albumin liver scintigraphy appears to be equally effective for selecting candidates for hepatectomy $(23,24)$. Findings of the current study have shown that Gd-EOB-DTPA-enhanced MRI findings correlated with total bilirubin values and ICG-R15, and that this modality has potential for use in the same manner as scintigraphy as a prognostic staging system and as a parameter for the preoperative assessment of hepatectomy. Shimizu et al (25) identified regional ischemic damage in the rat right hepatic lobe during the hepatic uptake phase of Gd-EOB-DTPA-enhanced MRI. The ability of Gd-EOB-DTPA-enhanced MRI to evaluate regional liver function might contribute to the preoperative assessment of hepatectomy. Tsuda et al (26) reported that the time to reach maximal enhancement and the half-life of such enhancement following Gd-EOB-DTPA injection are prolonged in the livers of rats with non-alcoholic steatohepatitis compared with the livers of rats with common fatty liver. Non-alcoholic steatohepatitis is now considered one of the most common types of chronic liver disease, which induces the development of liver cirrhosis and tumors (27-29). Although non-alcoholic steatohepatitis should be diagnosed early so that therapy begins early, the differences between non-alcoholic steatohepatitis and common fatty liver are not distinguishable by any radiological modality, including ultrasonography, computed tomography (CT) and MRI without contrast material $(30,31)$. Gd-EOB-DTPA-enhanced MRI has the potential to differentially diagnose human non-alcoholic steatohepatitis and common fatty liver. Our study results demonstrate that Gd-EOB-DTPA-enhanced MRI was capable of estimating liver function in humans similar to that observed in animal studies.

The gadolinium-diethylenetriaminepentaacetic (Gd-DTPA) derivative Gd-EOB-DTPA is comparable to Gd-DTPA in terms of being highly hydrophilic and water-soluble. In addition, a lipophilic ethoxybenzyl group enables selective intracellular uptake by hepatocytes $(2,18)$. Therefore, images of the early dynamic perfusion and late hepatocyte uptake phases can be captured following a single injection of Gd-EOB-DTPA $(1,2)$. The first phase within approximately $3 \mathrm{~min}$ following a bolus injection is equivalent to that of Gd-DTPA $(1,2)$. Focal lesions are more effectively identified using Gd-EOB-DTPA than contrast-enhanced dynamic-CT, with high diagnostic reliability and superiority (4-10). Although valid direct comparisons of Gd-DTPA and Gd-EOB-DTPA are rare, at least one study has proven that there are similar perfusion-phase tumor enhancement characteristics following the injection of the two contrast materials (2). Focal lesions with hepatocellular function, including focal nodular hyperplasia, adenoma and well-differentiated HCC, all absorb Gd-EOB-DTPA during the hepatocyte uptake phase. However, conditions without such hepatocellular function, including moderately or poorly differentiated HCC and liver metastases, do not absorb contrast material during the uptake phase (4-6,9). These imaging features are useful in characterizing focal lesions (4-6,9). Consequently, the early dynamic perfusion and late hepatocyte uptake phases are useful for detecting and characterizing focal hepatic lesions. In addition, Gd-EOB-DTPA has the same favorable safety profile as Gd-DTPA $(1,3,6,7,22)$. However, the delay in the hepatocyte uptake phase wastes more than $20 \mathrm{~min}(4,6,8-10)$. Certain investigators achieve the hepatocyte uptake phase at 10 and 20 min following injection $(4,8,9)$. The uptake rates of Gd-EOB-DTPA are similar at 10 and $20 \mathrm{~min}$ in 88 and $90 \%$ of focal nodular hyperplasia lesions, respectively (9). Huppertz et al (8) interpreted the enhancement characteristics of focal liver lesions at 10 and $20 \mathrm{~min}$ without a distinction in image quality. Differences in tumor-liver contrast-to-noise ratios in 23 liver metastases were not significant between 10 and $45 \mathrm{~min}$ following injection (4). The results of the present study suggest that a normally functioning liver uptakes considerable amounts of contrast material during the early period $(10 \mathrm{~min}$ ) following injection, which generates favorable contrast between focal lesions and the surrounding liver. Further studies are required to determine the image acquisition time appropriate to individual liver functions to decrease the duration required during the procedure. Once the time that is currently wasted obtaining the hepatocyte uptake phase image is shortened, Gd-EOB-DTPA-enhanced MRI may become as significant as ultrasonography, CT and MRI in the diagnosis of focal hepatic lesions (32-35).

This study has two limitations. Firstly, as the patient population comprised candidates for HCC therapy, no study subjects had extremely poor liver function (level $\mathrm{C}$ liver damage). Secondly, we did not observe Gd-EOB-DTPA washout from the liver. Although Gd-EOB-DTPA is absorbed by hepatocytes and excreted from the rat liver within approximately $60 \mathrm{~min}(16,17,26)$, these processes occur over $6 \mathrm{~h}$ in humans (1). The washout of Gd-EOB-DTPA is also prolonged in animals with a damaged liver $(16,17,26)$. Human patients would not be able to tolerate such protracted procedures.

In conclusion, the degree of liver enhancement with Gd-EOB-DTPA correlates with the level of liver function. Future clinical investigations are required to further evaluate the usefulness of Gd-EOB-DTPA-enhanced MRI as a test for diffuse liver disease and as a prognostic staging system for HCC. Decreasing the examination duration with rapid hepatocyte uptake phase images should also be investigated for patients with normal liver function.

\section{References}

1. Hamm B, Staks T, Muhler A, et al: Phase I clinical evaluation of Gd-EOB-DTPA as a hepatobiliary MR contrast agent: safety, pharmacokinetics, and MR imaging. Radiology 195: 785-792, 1995.

2. Vogl TJ, Kummel S, Hammerstingl R, et al: Liver tumors: comparison of MR imaging with Gd-EOB-DTPA and Gd-DTPA. Radiology 200: 59-67, 1996.

3. Reimer P, Rummeny EJ, Shamsi K, et al: Phase II clinical evaluation of Gd-EOB-DTPA: dose, safety aspects, and pulse sequence. Radiology 199: 177-183, 1996.

4. Reimer P, Rummeny EJ, Daldrup HE, et al: Enhancement characteristics of liver metastases, hepatocellular carcinomas, and hemangiomas with Gd-EOB-DTPA: preliminary results with dynamic MR imaging. Eur Radiol 7: 275-280, 1997.

5. Stern W, Schick F, Kopp AF, et al: Dynamic MR imaging of liver metastases with Gd-EOB-DTPA. Acta Radiol 41: 255-262, 2000. 
6. Huppertz A, Balzer T, Blakeborough A, et al: Improved detection of focal liver lesions at MR imaging: multicenter comparison of gadoxetic acid-enhanced MR images with intraoperative findings. Radiology 230: 266-275, 2004.

7. Bluemke DA, Sahani D, Amendola M, et al: Efficacy and safety of MR imaging with liver-specific contrast agent: U.S. multicenter phase III study. Radiology 237: 89-98, 2005.

8. Huppertz A, Haraida S, Kraus A, et al: Enhancement of focal liver lesions at gadoxetic acid-enhanced MR imaging: correlation with histopathologic findings and spiral CT - initial observations. Radiology 234: 468-478, 2005.

9. Zech CJ, Grazioli L, Breuer J, Reiser MF and Schoenberg SO Diagnostic performance and description of morphological features of focal nodular hyperplasia in Gd-EOB-DTPA-enhanced liver magnetic resonance imaging: results of a multicenter trial Invest Radiol 43: 504-511, 2008.

10. Hammerstingl R, Huppertz A, Breuer J, et al: Diagnostic efficacy of gadoxetic acid (Primovist)-enhanced MRI and spiral CT for a therapeutic strategy: comparison with intraoperative and histopathologic findings in focal liver lesions. Eur Radiol 18: 457-467, 2008.

11. Bollow M, Taupitz M, Hamm B, Staks T, Wolf KJ and Weinmann HJ: Gadolinium-ethoxybenzyl-DTPA as a hepatobiliary contrast agent for use in MR cholangiography: results of an in vivo phase-I clinical evaluation. Eur Radiol 7: 126-132, 1997.

12. Asbach P, Warmuth C, Stemmer A, et al: High spatial resolution T1-weighted MR imaging of liver and biliary tract during uptake phase of a hepatocyte-specific contrast medium. Invest Radiol 43 : $809-815,2008$

13. Clement O, Muhler A, Vexlar V, Berthezene Y and Brasch RC: Gadolinium-ethoxybenzyl-DTPA, a new liver-specific magnetic resonance contrast agent. Kinetic and enhancement patterns in normal and cholestatic rats. Invest Radiol 27: 612-619, 1992.

14. Muhler A, Freise CE, Kuwatsuru R, et al: Acute liver rejection: evaluation with cell-directed MR contrast agents in a rat transplantation model. Radiology 186: 139-146, 1993.

15. Muhler A, Heinzelmann I and Weinmann HJ: Elimination of gadolinium-ethoxybenzyl-DTPA in a rat model of severely impaired liver and kidney excretory function. An experimental study in rats. Invest Radiol 29: 213-216, 1994.

16. Schmitz SA, Muhler A, Wagner S and Wolf KJ: Functional hepatobiliary imaging with gadolinium-EOB-DTPA. A comparison of magnetic resonance imaging and 153gadolinium-EOB-DTPA scintigraphy in rats. Invest Radiol 31: 154-160, 1996.

17. Kim T, Murakami T, Hasuike Y, et al: Experimental hepatic dysfunction: evaluation by MRI with Gd-EOB-DTPA. J Magn Reson Imaging 7: 683-688, 1997.

18. Ryeom HK, Kim SH, Kim JY, et al: Quantitative evaluation of liver function with MRI using Gd-EOB-DTPA. Korean J Radiol 5: 231-239, 2004.

19. Makuuchi M, Kokudo N, Arii S, et al: Development of evidence-based clinical guidelines for the diagnosis and treatment of hepatocellular carcinoma in Japan. Hepatol Res 33: $37-51,2008$.

20. Clement O, Muhler A, Vexler VS, et al: Evaluation of radiation-induced liver injury with MR imaging: comparison of hepatocellular and reticuloendothelial contrast agents. Radiology 185: 163-168, 1992.
21. Kaplowitz N: Physiological significance of glutathione S-transferases. Am J Physiol 239: G439-G444, 1980.

22. Schuhmann-Giampieri G, Schmitt-Willich H, Press WR, Negishi C, Weinmann HJ and Speck U: Preclinical evaluation of Gd-EOB-DTPA as a contrast agent in MR imaging of the hepatobiliary system. Radiology 183: 59-64, 1992.

23. Kwon AH, Ha-Kawa SK, Uetsuji S, Inoue T, Matsui $Y$ and Kamiyama Y: Preoperative determination of the surgical procedure for hepatectomy using technetium-99m-galactosyl human serum albumin (99mTc-GSA) liver scintigraphy. Hepatology 25: 426-429, 1997.

24. Lau H, Man K, Fan ST, Yu WC, Lo CM and Wong J: Evaluation of preoperative hepatic function in patients with hepatocellular carcinoma undergoing hepatectomy. Br J Surg 84: 1255-1259, 1997.

25. Shimizu J, Dono K, Gotoh M, et al: Evaluation of regional liver function by gadolinium-EOB-DTPA-enhanced MR imaging. Dig Dis Sci 44: 1330-1337, 1999.

26. Tsuda N, Okada M and Murakami T: Potential of gadoliniumethoxybenzyl-diethylenetriamine pentaacetic acid (Gd-EOBDTPA) for differential diagnosis of nonalcoholic steatohepatitis and fatty liver in rats using magnetic resonance imaging. Invest Radiol 42: 242-247, 2007.

27. Ono M and Saibara T: Clinical features of nonalcoholic steatohepatitis in Japan: Evidence from the literature. J Gastroenterol 41: 725-732, 2006.

28. Shimada M, Hashimoto E, Taniai M, et al: Hepatocellular carcinoma in patients with non-alcoholic steatohepatitis. J Hepatol 37: 154-160, 2002.

29. Asanuma T, Ono M, Kubota K, et al: Super paramagnetic iron oxide MRI shows defective Kupffer cell uptake function in non-alcoholic fatty liver disease. Gut 59: 258-266, 2010.

30. Saadeh S, Younossi ZM, Remer EM, et al: The utility of radiological imaging in nonalcoholic fatty liver disease. Gastroenterology 123: 745-750, 2002.

31. Murata Y, Ogawa Y, Saibara T, et al: Tamoxifen-induced non-alcoholic steatohepatitis in patients with breast cancer: Determination of a suitable biopsy site for diagnosis. Oncol Rep 10: 97-100, 2003

32. Kubota K, Hisa N, Fujiwara Y, Fukumoto M, Yoshida D and Yoshida S: Evaluation of the intratumoral vasculature of hepatocellular carcinoma by power Doppler sonography: advantages and disadvantages versus conventional color Doppler sonography. Abdom Imaging 25: 172-178, 2000.

33. Kubota K, Hisa N, Nishikawa T, Ohnishi T, Ogawa Y and Yoshida S: The utility of tissue harmonic imaging in the liver: A comparison with conventional gray-scale sonography. Oncol Rep 7: 767-771, 2000.

34. Kubota K, Hisa N, Nishikawa T, et al: Evaluation of hepatocellular carcinoma after treatment with transcatheter arterial chemoembolization: comparison of Lipiodol-CT, power Doppler sonography, and dynamic MRI. Abdom Imaging 26: 184-190, 2001.

35. Kubota K, Yamanishi T, Itoh S, et al: Role of diffusion-weighted imaging in evaluating therapeutic efficacy after transcatheter arterial chemoembolization for hepatocellular carcinoma. Oncol Rep 24: 727-732, 2010. 